Texture of Crystalline Solids, 1980, Vol. 4, pp. 41-56

0309-7951/80/0401-0041\$04.50/0

() 1980 Gordon and Breach, Science Publishers, Inc.

Printed in the United States of America

\title{
LATTICE CURVATURES PRODUCED BY THE HEAVY DEFORMATION OF POLYCRYSTALS *
}

\section{L. DILLAMORE}

Department of Metallurgy and Materials, University of Aston, Birmingham, England

(Received October 15, 1979)

\begin{abstract}
An outline nomenclature is given for some of the microstructural heterogeneities found in heavily deformed single phase cubic metals. The relationship of these features to the development of lattice curvatures is discussed and the importance of lattice curvatures in relating deformation and annealing textures is assessed.
\end{abstract}

\section{INTRODUCTION}

In 1965 the American Society for Metals organised a seminar on Recrystallisation, Grain Growth and Textures ${ }^{1}$ and, quite properly, included among the papers presented at that meeting one concerned with "The Nature of the cold Worked state." In so doing they acknowledged that the microstructure resulting from deformation is important in influencing the kinetics of recrystallisation and the evolution of the recrystallised microstructure. The paper actually presented ${ }^{2}$ unfortunately had little relevance to the rest of the conference, being concerned with very low strain deformation of single crystals, and low density dislocation structures which would not have been likely to cause recrystallisation in annealing. However, the intention was worthy.

This conference may be considered as the first (although the vagaries of subsequent numbering require that it be regarded as the zeroth) in a series of international conferences concerned with texture in materials.

* Based on a paper given at the 10th Conference on Materials Testing in Metallurgy at Balatonaliga, Hungary, May 1979, organised by The Hungarian Mining and Metallurgical Society and the Research Institute for Ferrous Metallurgy in cooperation with the Hungarian Academy of Sciences \& Bergakademie Freiberg (G.D.R.). 
The next one (the first) held in Clausthal in $1968^{3}$ gave little attention to microstructures in the deformed state, but a paper presented at the following one (the second) ${ }^{4}$ in Krakow gave an outline analysis of the interaction between texture development and microstructural evolution during deformation and this was expanded in one of the papers presented at the third conference in the series at Pont-a-Mousson in $1973^{5}$ when it was elaborated to draw some inferences relevant to annealing texture development. The fourth conference in Cambridge in $1975^{6}$ again ignored the subject but there was a large body consisting of both analytical and experimental studies of the structure of heavily deformed metals in the fifth conference in Aachen in $1978^{3}$ and the literature on the subject is now growing rapidly, e.g. Ref. 8 .

The aim here is to review recent progress in our knowledge of the structure of heavily deformed polycrystalline metals. In this task it is not helpful that there are no agreed terms to describe the features that occur and more work is needed to understand how they arise before an unambiguous terminology can develop. Terms such as slip bands, shear bands, transition bands and deformation bands are in common but ill-defined use. At the outset it seems worthwhile to give some definitions of these, and other terms, as they will be used here, as a contribution to the developing debate on nomenclature. This particular set of definitions will be based upon visual information, orientation variables and, in some cases, on presumptions concerning the mode of formation.

\section{NOMENCLATURE}

1. Deformation bands. This is a term that may be used to cover a wide range of microstructural heterogeneities, that are the result of plastic deformation. In its broadest sense it could include deformation twins, e.g. Neumann bands, or regions containing fine twins or stacking faults in low stacking fault energy f.c.c. metals. It may more commonly be recognised as a region where the strain state deviates from that of the surroundings or be revealed by variations in orientation (as in the examples shown in Figure 1) or in dislocation density. Some deformation bands have more specific names, which are given in the following, for others there is no clear and generally acceptable terminology.

2. Slip bands. These are best seen in single crystals, on which they are revealed, on previously polished surfaces, as slip steps which are crystallographically consistent with the traces of known slip planes. There are various kinds of slip bands, such as: bands of fine slip, bands of secondary slip, etc. Bands of wavy slip have often been reported and assumed to be due to cross-slip, as indeed they may be. It is, however, possible to have an appearance of wavy slip arising from other modes of deformation than simple slip. Some examples of slip bands are shown in Figure 2 . 


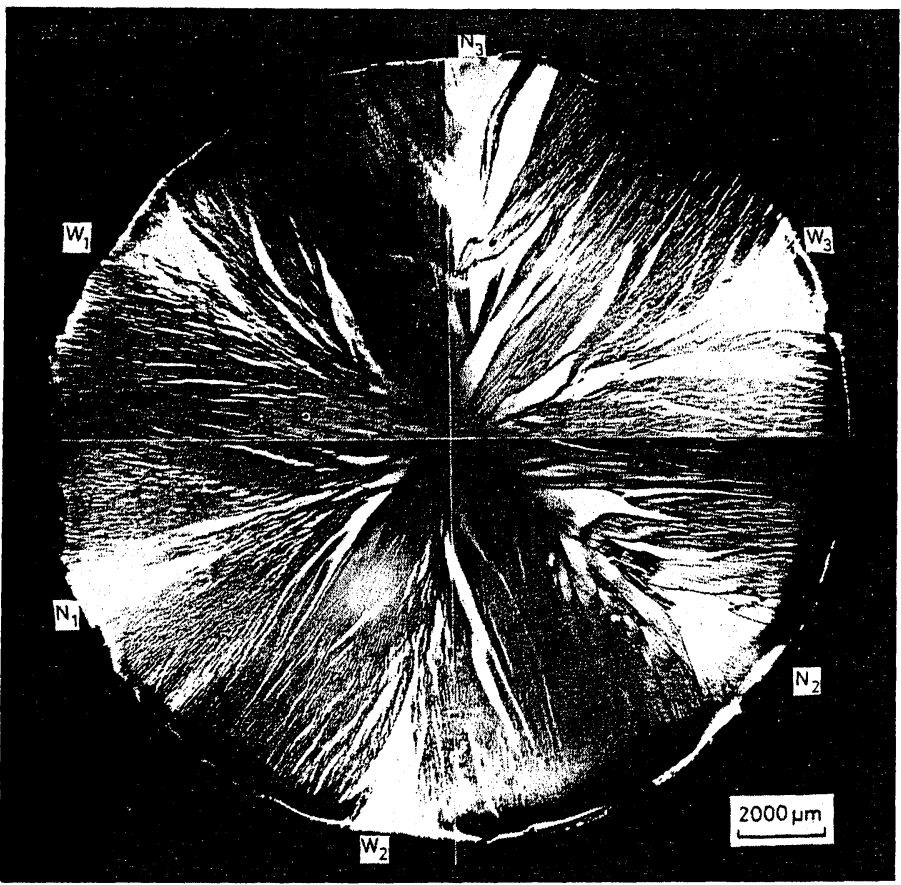

Figure 1. Pattern of deformation bands on a cross section of a twisted <lll> aluminium crystal. (Kreisler \& Doherty ${ }^{9}$ )

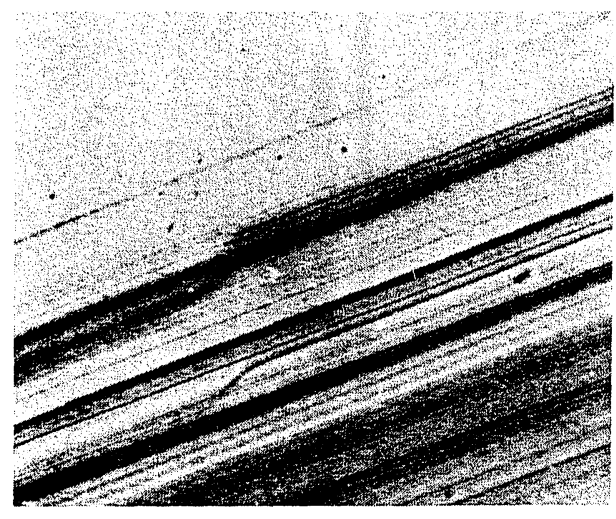

(a)

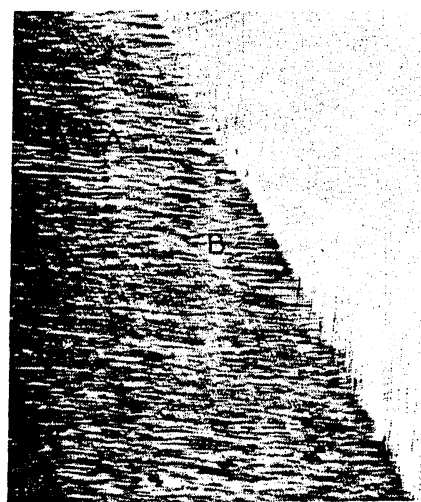

(b)

Figure 2. (a) Slip bands of clustered fine slip in an aluminium crystal deformed $30 \%$ at room temperature (Van Bueren ${ }^{10}$ ). (b) Complex slip bands, showing wavy slip, multiple slip and single slip on a twisted <lll> aluminium crystal (Kreisler \& Doherty ${ }^{9}$ ).

3. Kink bands. This is a term used by orowan ${ }^{11}$ for a region in which the crystal orientation varies so that in crossing the band the orientation difference from the surrounding material increases to a maximum and then reduces to zero. 
The nature of a kink band is most readily indicated by the deformed single crystal shown in Figure $3 a$, but the kinking nature of inhomogeneous deformation may be inferred on metallographic sections by using orientation sensitive etching agents (Figure $3 \mathrm{~b}$ ). It is the kink lattice structure which is most important here, although it will usually be the result of inhomogeneous strains of the type shown in Figure $3 a$.

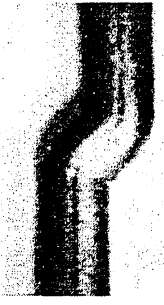

(a)

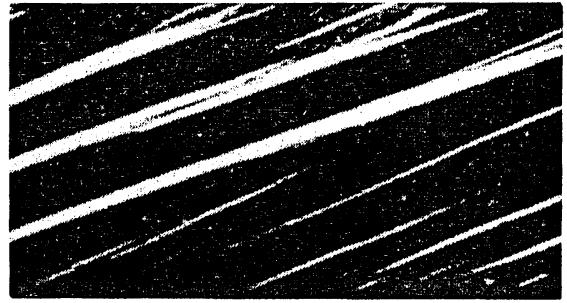

(b)

Figure 3. (a) A kink in a zinc crystal (Barrett ${ }^{2}$ ).

(b) Probable kink bands in a deformed aluminium crystal (Karduck, Gottstein \& Mecking ${ }^{13}$ ).

4. Shear bands. The name of these features implies a mechanism of formation and does not necessarily define their structural features other than their shape. If, as implied by the name, the strain state in these bands is simple shear, the shear plane will lie parallel to the composition plane between the band and the matrix if the strain in the matrix is zero. In this case, the orientation across the shear band will in general vary as for a kink band.

There are some exceptions to this, as when the shear plane is a simple slip plane-the shear band is then a slip band-or for a few special cases for which the shear strain state is irrotational. The latter case may have the appearance of a band of wavy slip but the "slip lines" may not be the result of cross-slip. The best evidence for shear bands is obtained from the displacement field, as revealed by fiducial markings (Figure 4 a) but inferences may be drawn from polished edge sections, metallographic sections and electron micrographs (Figures $4 \mathrm{~b}, \mathrm{c}$, d respectively) that shear banding has occurred.

5. Microbands. This term has been used by Grewen and co-workers ${ }^{16}$ for a feature found in cubic metals. An example is shown in Figure 5. The bands consist of parallel dislocation arrays, in section appearing like tram-lines, and these arrays are said to lie initially in (lll) planes in f.c.c. metals and (110) planes in b.c.c. metals. These microbands are first found at about $10 \%$ strain in rolled metals and increase in density and rotate into the rolling plane with increasing strain, thus becoming dissociated from the crystallographic planes on which they form.

There are a number of possibilities for the formation of microbands. Some workers ${ }^{16}$ regard them as micro-shear bands, which seems unlikely if the band plane is a slip plane 


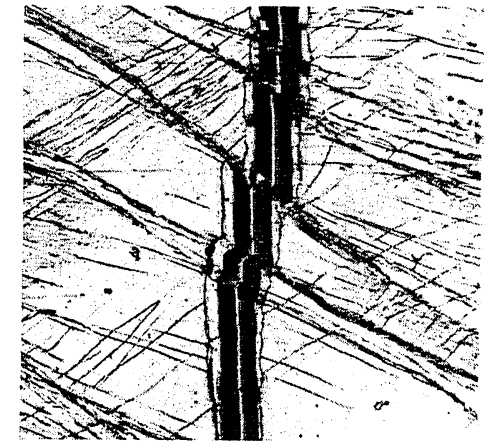

(a)

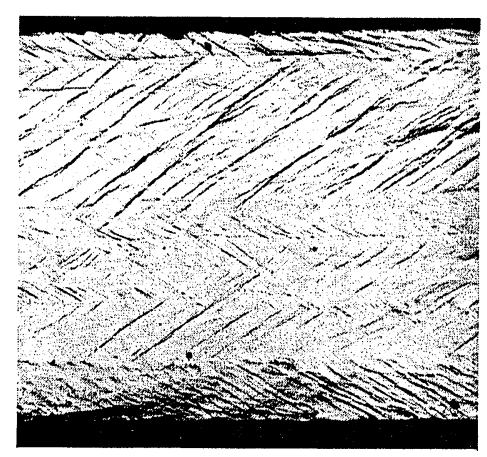

(b)

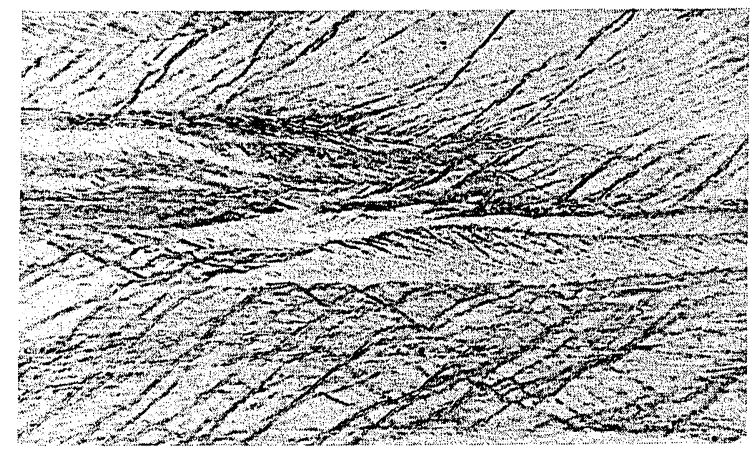

(c)

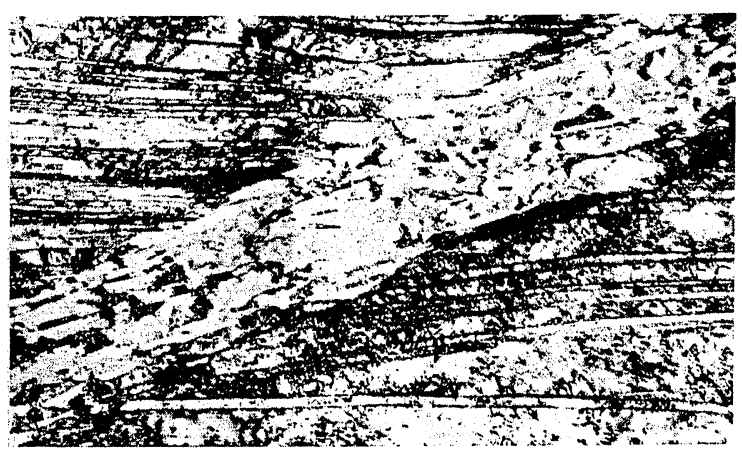

(d)

Figure 4. (a) Shear band displacements revealed by an intersecting groove. (b) Shear band markings shown on a polished edge section of a $\mathrm{Al}-4 \% \mathrm{Cu}$ alloy rolled $70 \%$ (Dillamore, Roberts \& Bush ${ }^{14}$ ). (C) Shear bands revealed metallographically on a longitudinal section of $70 / 30$ brass rolled 60\%. (d) Electron microstructure corresponding to a shear band in an austenitic strainless steel rolled 65\% (Blicharski and Gorczyca ${ }^{15}$ ).

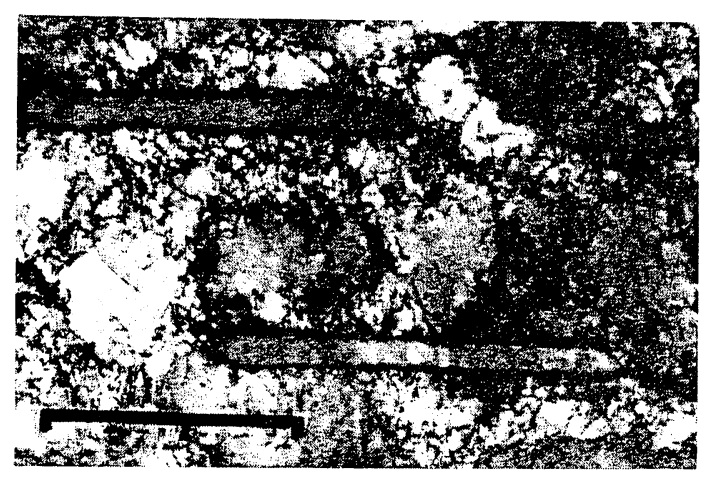

Figure 5. Microbands in copper rolled 20\% (Grewen, Hüber \& Hather $\left.1 y^{16}\right)$. 
since a shear on a slip plane causes no misorientation across that plane, while the evidence that the bands rotate with the macroscopic deformation implies that the band boundaries define a developing lattice curvature. They may alternatively be the first signs in the electron microstructure of the nucleation of a curvature. Their initial orientation may then be explained by detailed arguments advanced previously. ${ }^{17}$ A third possibility is that they represent a macro-kink band formed because patches of dislocation debris lying on the most active slip plane impede intersecting slip. This would conveniently explain why they have the appearance of tram-lines, since it would require two patches of debris to isolate a region of the material, a single patch would create a quite different feature.

6. Transition bands. This term developed from the work of $\mathrm{Hu}^{1 \dot{8}}$ (who called them microbands) and Walter and Koch 9 to describe a structure observed in the electron microscope, and illustrated in Figure 6 , in which elongated cells are found arranged so that the misorientations across successive cell walls are cumulative. The structure thus defines a region of lattice curvature linking two dissimilar orientations. A possible configuration is shown in Figure $7 \mathrm{a}$ in which the continuous change in orientation of one crystal plane is mapped in space. Two such configurations back-to-back would form a kink band (Figure $7 \mathrm{~b}$ ).

Relating the electron microstructure to that seen in the light microscope, a transition band may thus be regarded as a deformation band boundary across which there is an orientation change. The transition band achieves more gradually the change in orientation that is found across a grain boundary.

\section{THE OBSERVATION OF INHOMOGENEOUS DEFORMATION}

The description of different features given above has relied heavily on the methods of observation used in their study. All of these methods give information which is essentially two dimensional and the fine scale of the occurrence of the various bands makes it difficult to use two surface or three surface analysis to define their characteristics. The two dimensional information contained in Figures $1-6$ is of several different types. The slip band markings and the fiducial marks give partial insight into the displacements occurring, indicating where strain has been concentrated and where there are deviations in the strain state. This does not reveal very much about the structure developed by such concentrated or deviant strain. Observations using optical metallography are related to structure; an etchant may show qualitatively that there are orientation differences from point to point and it may be sensitive to dislocation density and distribution. There may also be evidence of displacements that have been produced. None of this information is detailed or specific, but it does provide a link between the surface observations of displacement fields and the detailed information that can be obtained in the electron microscope. This can show dislocation distributions and crystallite orientations, 

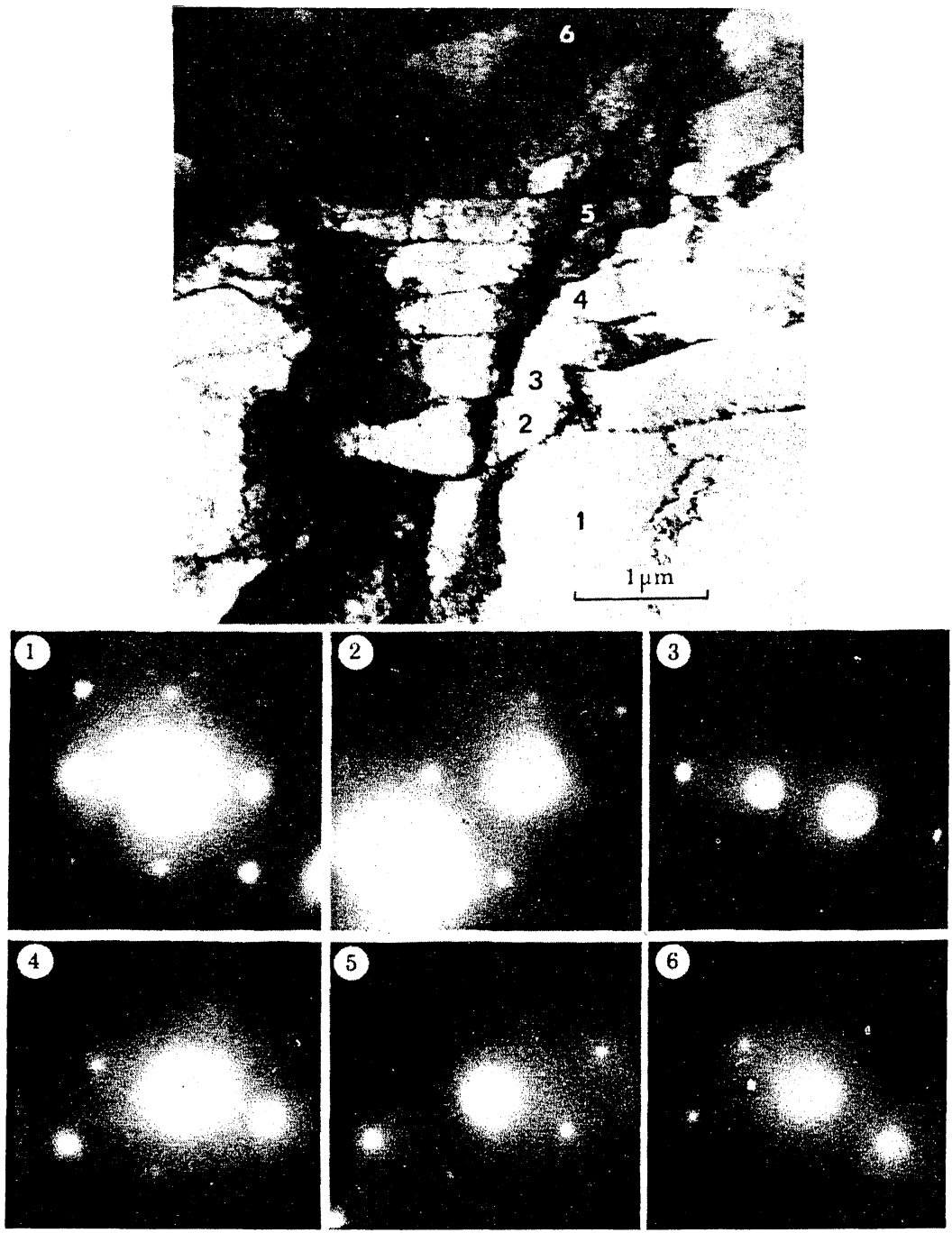

Figure 6. Elongated subgrain structure and associated diffraction patterns corresponding to a transition band with a $30^{\circ}$ rotation about the beam direction (Dillamore, Morris, Smith \& Hutchinson ${ }^{20}$ ).

but both are needed in order to characterise the structure, because it cannot be assumed that a particular subgrain morphology is indicative of the existence or absence of a long range lattice curvature.

It is tempting to suppose that ribbon-like subgrain morphology does correspond with a curved lattice and this will usually be so, but in the formation of shear band it is, as previously stated, not necessary for kinking to occur, yet it seems possible that even then flow parallel to a shear plane will produce a dislocation distribution which is elongated in the direction of flow. 


\section{(a) TRANSITION BANDS}

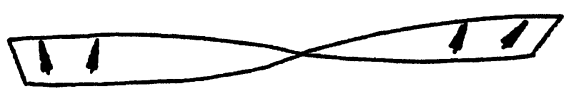

mainly

twist

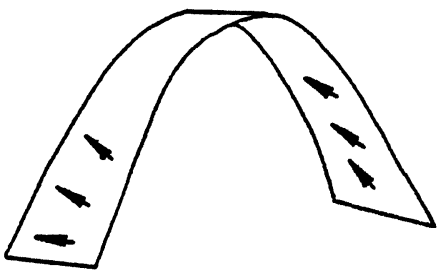

mainly

tilt

(b) KINK BANDS
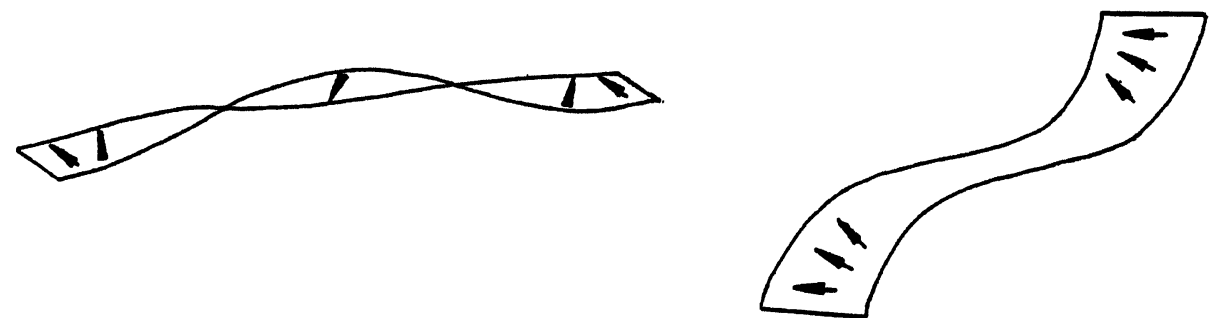

Figure 7. Representation of lattice curvatures by the bending of a single crystal plane. The arrow heads all represent a single crystal direction.

The information derived from the three types of observation is all required if the mechanisms of formation of the microstructure are to be understood. From the viewpoint of relating deformation structure to the annealing response, however, the displacement fields that have produced the microstructure are not important. Of first importance are the distributions of dislocations, which both constitute the sources of the energy to drive the annealing processes and define the lattice curvatures.

There is a need for much more work, using HVEM and STEM, to characterise the deformation structure at high strains but there is also a need for an analysis of the formation of such structures. In the next section progress in this direction is outlined.

THE DEVELOPMENT OF STRUCTURE IN DEFORMATION

Understanding of the behaviour of polycrystals undergoing deformation has not advanced very greatly since Taylor ${ }^{21}$ proposed his homogeneous deformation theory. The exact theories of the elastic behaviour of polycrystals of Kröner ${ }^{2}$ and Kneer $^{3}$ do in principle permit their behaviour under 
elasto-plastic conditions to be computed, provided that complete information of the effect of strain history on the yield criterion of a crystal is known. Berveiller and $\mathrm{Zaoui}^{24}$ are working on this problem and are able, by making some assumptions about yield behaviour, to improve the quantitative accuracy of theories of texture development. The net effect of the interactions between crystals in the polycrystal is to increase the spread in the texture. However, this statistical result is only slightly informative about the development of microstructure.

The nucleation of deformation banding in polycrystals is known to occur most prolifically at low strains and to diminish with increasing strain. This, and the fact that deformation bands are more prevalent in coarse than in fine grained polycrystals, is to be expected as a consequence of the compatibility and equilibrium conditions. Precisely how the inhomogeneous deformation develops will depend for a given grain on its orientation and the orientations and positions of its neighbours. Initially the strain state may vary continuously from point to point or the strain may divide between different regions separated by planes in which the strains match, but progressively with strain the distinction between these two will diminish. This is because in a continuously varying strain state the relative rotations of different regions are not a simple function of differences in strain state.

Because dislocations are necessary to define misorientations, the dislocation configurations which develop will be a consequence primarily of differences in orientation, the geometrically redundant dislocations contain additional information about strain state differences. Thus surfaces, possibly curved, will develop which define locations of similar orientation. Further increments of strain give rise to differences in strain that will depend, at least partly, on orientation and, in general, the surfaces of iso-orientation will gradually become more polygonal, i.e. be composed of segments which tend to become planar.

The dislocation walls that are present in order to define misorientations must, unless the misorientations are removed by subsequent straining, be rotated in response to the macroscopic shape change imposed by deformation. Thus in rolling the structure will become progressively more flattened and elongated, so that the curvatures are represented by interleaved ribbon-like subgrains; in axi-symmetric compression the structure will be of disc shaped subgrains and in wire-drawing the cell boundaries will be elongated parallel to the wire axis.

There are other causes of inhomogeneous deformation than grain interactions in polycrystals; the imposed shape change may not permit homogeneity, or surface friction effects may impose gradients of stress and strain. A further cause that is intrinsic to the material is found when a load instability occurs. The incidence of shear banding in rolling is the result of a load drop ${ }^{4}$ as also is the occurrence of the deformation bands in drawn [100] copper crystal observed by 
Ahlborn ${ }^{25}$ (Figure 8). Except for materials with a sharp yield point, such instabilities and the resultant inhomogeneities

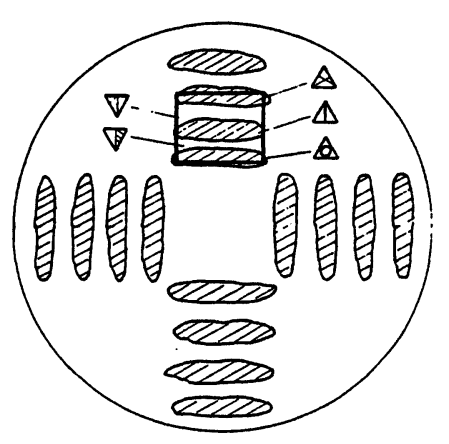

(a)

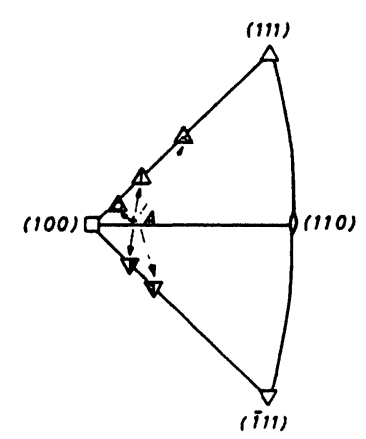

(b)

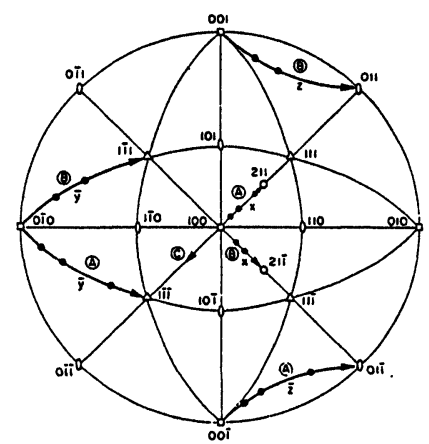

(c)

Figure 8. (a) Schematic representation of deformation bands in drawn <100> oriented copper crystal (from Ahlborn ${ }^{25}$ ). (b) Orientation determinations corresponding to (a). (c) Analysis of deformation banding of (a) (after Chin and Wonsiewicz ${ }^{28}$ ).

are found after strains of $40 \%$ or more, and they thus represent a systematically occurring heterogeneity that develops when the texture is substantially formed. They are different in this from the inhomogeneities formed at low strain, which can only be regarded as causing scattering of orientations. Such scattering is however influential in the development of microstructure.

\section{AXISYMMETRIC DEFORMATION OF CUBIC METALS}

The developments of lattice curvatures from heterogeneities formed at low and at high strains will be illustrated by reference to the compressive deformation of iron 26,27 and to the results of Ahlborn ${ }^{25}$ and their analysis by Chin and Wonsiewicz ${ }^{2}$ concerning the drawn [100] crystals of copper. Axisymmetric deformation is easier to describe than deformation by rolling but the principles are similar.

Consider the crystal rotation fields indicated in Figure 9 for compression in b.c.c. metals and tension in f.c.c. metals. In earlier work ${ }^{27}$ it was assumed that lattice curvatures occur by small deviations in orientation developing gradually and it was explained how this could give curvatures linking [100] and [111] along the [0II] zone. This may be recognised by reference to the dashed line linking [4II] and [110], which is a divergent line, material below this line rotating to [100], material above it rotating to [111]. What cannot be seen in Figure 9 is that the rotation about the axial direction is dependent on the path followed to the end point, so that gradual divergence in orientation can also occur by the formation of curvatures around the axial direction. The diagram shown in Figure 10 is an attempt to illustrate 


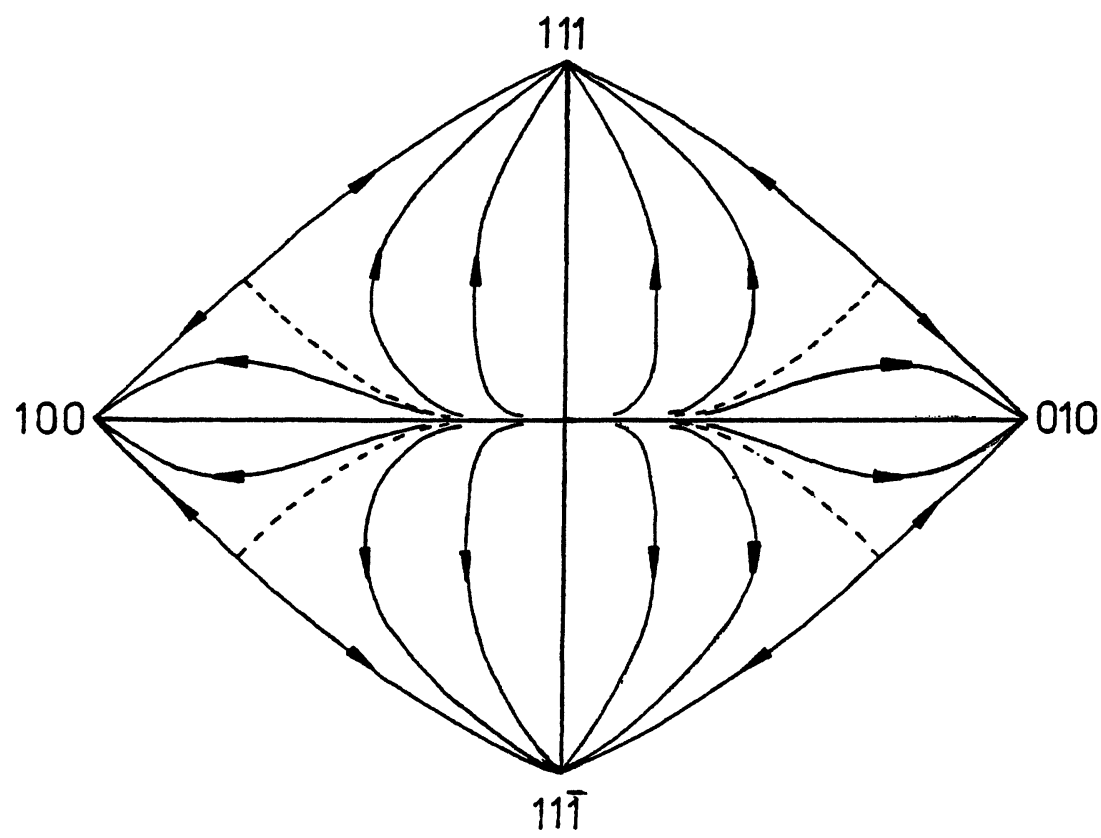

Figure 9. Crystal rotations for axisymmetric deformation -compressive for b.c.c. metals, tensile for f.c.c. metals-for axial directions in four unit triangles.

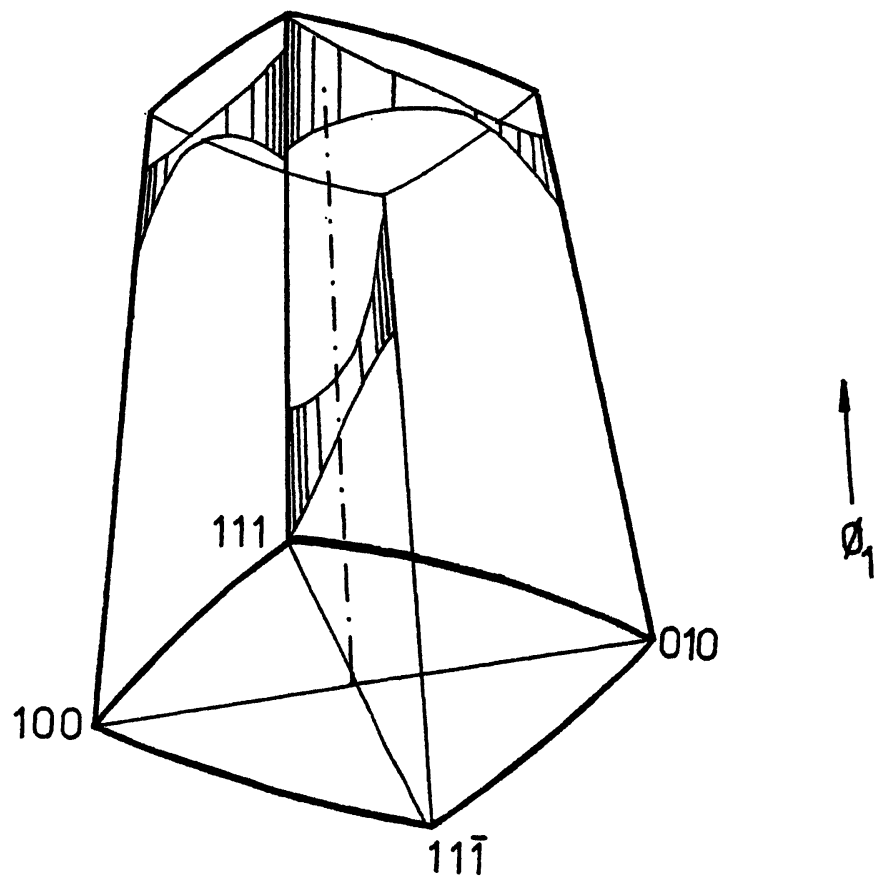

Figure 10. Portion of orientation space relating to Figure 2 but showing the dimension which represents rotation about the fibre axis. [111] is the nearest point in this perspective view. 
this. It is a perspective drawing of a prism constructed perpendicular to the four unit triangles of Figure 9. Distance from the base indicates the angular rotation about the axial direction ( $\Phi_{1}$ in Bunge's notation). The band drawn in the face joining [111] and [100] represents the spread of orientation, the hatching represents the variation in orientation density, that may develop from a grain that lay initially close to the [411] - [110] divergent line. The curvatures are not along a line or orientation space, but in a curved plane. There will, as part of the structure, be regions of partial [100] and [111] fibre texture, as well as a transition zone linking the two. The sub-boundary character - the grains being pancaked-will vary from mainly [100] twist-boundary to mainly [111] twist boundary, via [01I] tilt boundary.

If in addition to the gradual evolution of curvatures the formation of deformation bands occurs, then the deformation band boundaries can contain marked curvatures, and rotations quite different from those in Figure 9 can occur. This is clearly so in the data shown in Figure 8 , where rotations away from [100] to the four <Ill> occur, contrary to the trends shown in Figure 9. The unpredicted rotation will continue only so long as the inhomogeneous deformation continues, but by this time a marked spread of orientation is developed. In the case of the set of deformation bands analysed in detail in Figure 8 , a return of homogeneous straining would cause the curvatures to develop to link [11i] via [100] to [111].

In general a deformation band might create a curvature joining points in orientation space at some distance from each other, and in the modes of deformation relevant to Figure 9 curvatures linking [100] - [111], [111] - [010], [010]-[1II], [1II] - [100], [111] - [1II] or, less probably, [100] - [010] can be envisaged. In complex cases, several or all of these curvatures could exist together and there are, of course, others to be added if the space occupied by [100], [III] and [111] are included in the picture. In Figure 10 two of the $\langle 100\rangle-\langle 111\rangle$ curvatures are illustrated, together with [1II] - [11], which shows two <lll> partial fibre textures linked by a spread which includes some [110] orientation. Deformation banding linking the first and third (or second and fourth) quadrants of Figure 9 would take up this orientation spread.

\section{LATTICE CURVATURES AND THE NUCLEATION} OF RECRYSTALLISATION

There is much good evidence that the nucleation of recrystallisation occurs readily, and in some cases predominantly, in deformation band boundaries.18-20 This is evidenced in the scanning electron micrograph of Figure 11. 29 The identification of those orientations that are to be found within these boundary regions thus provides one mechanistic link between the texture formed in deformation and in annealing. There are other sites for the nucleation of recrystallisation and some discussion of the orientation dependence of 


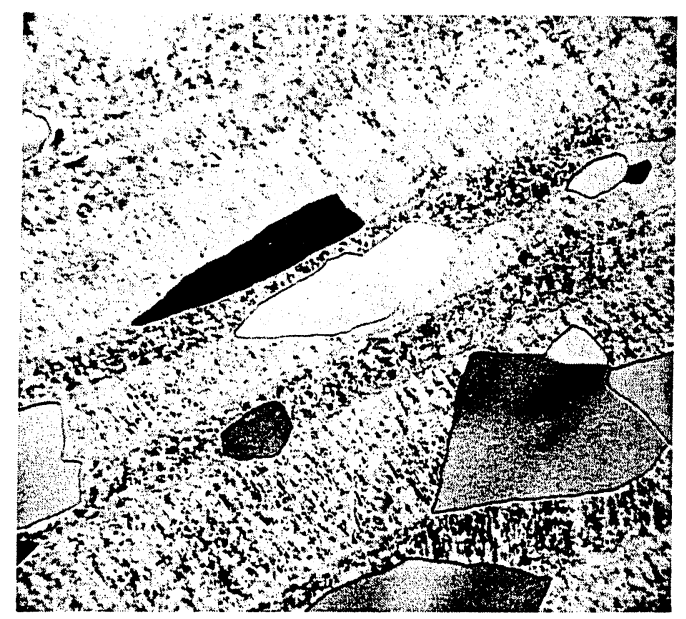

Figure 11. Scanning electron micrograph showing recrystallised grains in deformation band boundaries. $(100)[001] \mathrm{Fe}-3 \% \mathrm{Si}$ crystal rolled $60 \%$ and annealed at $600^{\circ} \mathrm{C}$.

these have been given in earlier work. 27-30 However, it is worthwhile to examine for the case of compression whether there is any relationship between the curvatures that have been defined here and the annealing texture components. This may be done using data from the literature ${ }^{27}$ and reproduced in Figure 12.

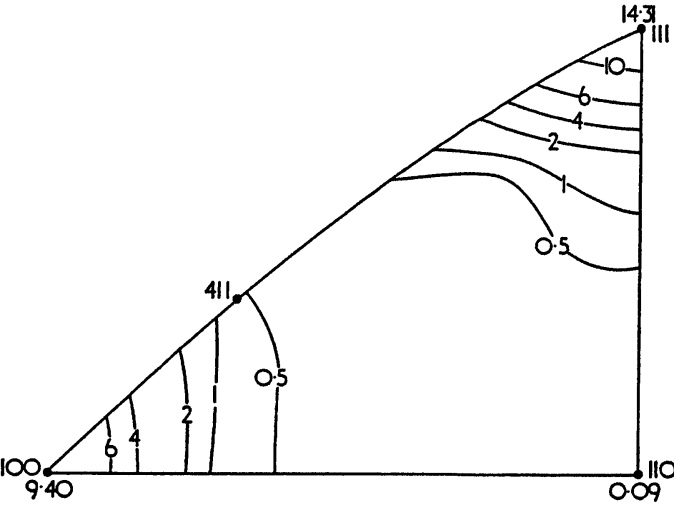

(a)

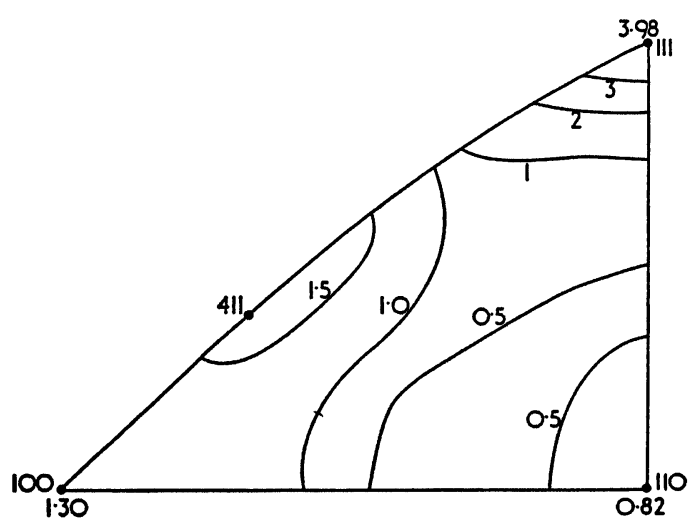

(b)

Figure 12. (a) Compression texture of an Fe $0.02 \% \mathrm{C}$ alloy deformed $87.7 \%$ and (b) annealed $2 \mathrm{~min}$ at $700^{\circ} \mathrm{C}$.

The texture of iron compressed $88 \%$ is shown in Figure $12 \mathrm{a}$ and the texture after 2 minutes at $700^{\circ} \mathrm{C}$ is shown in Figure $12 \mathrm{~b}$. The deformation texture qualitatively accords with the expectations resulting from the rotations of Figure 9 but before turning to the annealing texture it is worthwhile 
to summarise where the curvatures resulting from these rotations occur.

The most obvious curvature links [100] - [111] along the [0II] zone; the maximum rate of change of orientation will lie about $20-25^{\circ}$ from [100] for this spread. There will be some curvatures linking [111] and [11I], centred on [110] and, not to be overlooked, there will be curvatures around the [100] and [111] fibre axes. The spreads of orientation considered could each be present within the volume occupied by one grain of the starting material. The annealing texture has components centred on [100], [111], [110] and a position about 20-25 from [100] in the [01I] zone (near [311]). In an earlier paper ${ }^{27}$ the [100] and [111] components were ascribec to an in-situ mechanism of subgrain growth in regions of rather uniform orientation, but, as has now been amplified, they could be the result of transition band nucleation.

If the hypothesis that nucleation is predominantly from curvatures is correct, it should be possible to obtain a further understanding of the quantitative features of the annealing texture. Clearly nucleation in curvatures gives ample scope for spread in the annealing texture but focussing on the centre of each component the effectiveness of each nucleation site may be assessed by comparing the intensity in the deformation texture with that in the annealing texture. This is done in Table $I$.

TABLE I

\begin{tabular}{cccc} 
Orientation & $\begin{array}{c}\text { Intensity in } \\
\text { Deformed State } \\
I_{\mathrm{D}}\end{array}$ & $\begin{array}{c}\text { Intensity in } \\
\text { Annealed State }\end{array}$ & $\begin{array}{c}\text { Ratio } \\
I_{\mathrm{A}} / I_{\mathrm{D}}\end{array}$ \\
\hline$[100]$ & 9.40 & 1.30 & .14 \\
{$[111]$} & 14.31 & 3.98 & .28 \\
{$[110]$} & 0.09 & 0.82 & 9 \\
{$[311]$} & 0.40 & 1.60 & 4
\end{tabular}

In an analysis of the conditions for nucleation in transition bands ${ }^{20}$ it was suggested that the factors of importance are the sharpness of the curvature and the stored energy available to support the growth of a nucleus. The curvature should be neither too gentle, nor too sharp and it is advantageous if a gradient of stored energy exists across a transition band such that there is an increasing driving force as growth proceeds in the early stages of development of the nucleus.

Against these considerations the [100] and [111] twist bands would not have a gradient of stored energy but the [111] has on average a more sharply developed curvature than [100] and a higher level of stored energy. The [110] and near [311] orientations are from sharply curved transition regions, the angular range being $70^{\circ}$ for the [110] and $55^{\circ}$ for the [311]. The stored energy distribution across the bands containing 
[110] will be quite uniform and at a level similar to that of the [111] twist band. There is expected to be a gradient through [311] linking low energy [100] and high energy [111] regions.

It is not reasonable to compare the curvatures, [100] and [111], which are part of the stable texture components with those, [110] and [311], which are in divergent regions, since the intensity of the latter components in the deformation texture will be exclusively within transition bands while the [100] and [111] intensities will include regions of uniform orientation. The effectiveness of [100] and [111] in generating recrystallisation nuclei can be compared, as can the effectiveness of [110] and [311]. The ratio $I_{A}: I_{D}$ gives some measure of this. In both cases the greater effectiveness is found for the orientation which has the larger curvature associated with it, coupled with a higher level of stored energy.

other factors, related for instance to growth, need to be considered but there are nevertheless indications of the importance of lattice curvatures in contributing to the nucleation of recrystallisation. Completely similar arguments can be advanced for rolling textures which also contain curvatures both within the stable texture components and linking them across divergent planes. ${ }^{30}$ It will, however, require considerably more experimental work to ascertain that the curvatures are as important as has been inferred here.

It may prove necessary to distinguish transition bands and kink bands in respect of their effectiveness as nucleation sites. A single transition band may be more effective, not in the sense that it provides nuclei more prolifically but in that the kink band has two separate regions of similar orientation, so that impingement will occur early in growth from the nucleus. Less prolific nucleation at the centre of the kink band may produce larger nuclei than those formed in the sharp transition zones. These could then, as pointed out previously, ${ }^{31}$ give rise to annealing textures that are similar to the deformation textures but by a mechanism quite different from the in-situ one usually considered.

\section{CONCLUDING OBSERVATIONS}

The foregoing has concentrated on examining the types of lattice curvature that may arise in single phase cubic metals and has excluded any consideration of the influence of twinning and second phase particles. In other work 1 it has been concluded that fine twinning in low stacking fault energy materials is most important as a source of driving energy to sustain the growth of nuclei formed in the way that has been considered here, but growth twinning must also be considered in such materials as a mechanism for generating new orientations in the annealing texture.

Humphreys ${ }^{3}, 33$ has considered the effect of second phase particles in generating lattice curvatures and his ideas are quite close in character to those discussed here. There is room for much further study of this subject, examining in particular the effects of inclusion shape, coherence and deformability. 
The evaluation of the possible influence of lattice curvatures, formed during deformation, on the development of annealing textures reinforces the views expressed previously of their importance.

\section{ACKNOWLEDGEMENTS}

I wish to thank Dr. W. B. Hutchinson for invaluable discussions, advice and for permission to use unpublished information.

\section{REFERENCES}

1. Recrystallisation, Grain Growth and Textures (ASM, Metals Park, Ohio, 1965).

2. S. J. Basinski and z. S. Basinski, in ref. 1, p. 1.

3. J. Grewen and G. Wassermann, eds., Textures in Research and Practice (Springer-Verlag, Berlin, 1969).

4. Quantitative Analysis of Texture (Polish Academy of Sciences, Krakow, 1971).

5. Proc. 3rd European Colloquium on Textures, Pont-a-Mousson, 1973.

6. G. J. Davies, I. L. Dillamore, R. Hudd and J. S. Kallend, eds., Textures and the Properties of Materials (Metals Society, London, 1975).

7. G. Gottstein and K. Likker, eds., Textures of Materials (SpringerVerlag, Berlin, 1978).

8. Metals Forum, published by the Australasian Inst. of Metals, Vol. 1, No. 3, September, 1978.

9. A. Kreisler and R. D. Doherty, Metals Science, 12, 551 (1978).

10. H. G. Van Bueren, Imperfections in Crystals (North-Holland, Amsterdam, 1960).

11. E. Orowan, Nature, 149, 643 (1942).

12. C. S. Barrett, Structure of Metals, 2nd Ed. (McGraw-Hill, New York, 1952), p. 375.

13. P. Karduck, G. Gottstein and H. Mecking, in ref. 7, p. 377.

14. I. L. Dillamore, J. G. Roberts and A. C. Bush, Metal Science, 13, 73 (1979).

15. M. Blicharski and S. Gorcyzca, Metal Science, 12, 303 (1978).

16. J. Grewen, J. Huber and M. Hatherly, in ref. 8, p. 115.

17. I. L. Dillamore, in ref. 7, p. 67.

18. H. Hu, in Recovery and Recrystallization of Metals, H. Himel, ed. (Interscience, New York, 1963), p. 311

19. J. L. Walter and E. F. Kock, Acta Met., 11, 923 (1963).

20. I. I. Dillamore, P. L. Morris, C. J. E. Smith and W. B. Hutchinson, Proc. Roy. SOC., A329, 405 (1972).

21. G. I. Taylor, J. Inst. Met., 62, 307 (1938).

22. E. Kröner, Acta Met., 9, 155 (1961).

23. G. Kneer, Phys. Stat. Sol., 9, 825 (1965).

24. M. Berveiller and S. Zaoui, in ref. 7, p. 319.

25. A. Ahlborn, in ref. 1, p. 374.

26. Y. Inokuti and R. D. Doherty, Acta Met., 26, 61 (1978).

27. I. L. Dillamore, H. Katoh and K. Haslam, Texture, 1, 151 (1974).

28. G. Y. Chin and B. Wonsiewicz, Trans. TMS-AIME, 245, 871 (1969).

29. W. B. Hutchinson, University of Aston, unpublished work.

30. I. L. Dillamore and H. Katoh, Metal Science, 8,73 (1974).

31. I. L. Dillamore, in ref. 8, p. 136.

32. F. J. Humphreys, in ref. 8, p. 123.

33. F. J. Humphreys, Metal Science, 13, 136 (1979). 